\title{
High-Energy Proton-Proton Forward Scattering and Derivative Analyticity Relations
}

\author{
R. F. Ávila ${ }^{1}$, E. G. S. Luna ${ }^{2}$, and M. J. Menon ${ }^{2}$ \\ ${ }^{1}$ Instituto de Matemática, Estatística e Computação Científica \\ Universidade Estadual de Campinas, UNICAMP \\ 13083-970 Campinas, SP, Brazil \\ ${ }^{2}$ Instituto de Física Gleb Wataghin \\ Universidade Estadual de Campinas, UNICAMP \\ 13083-970 Campinas, SP, Brazil
}

Received on 15 May, 2001

\begin{abstract}
We present the results of several parametrizations to two different ensemble of data on $p p$ total cross sections $\left(\sigma_{t o t}^{p p}\right)$ at the highest center-of-mass energies (including cosmic-ray information). The results are statistically consistent with two distinct scenarios at high energies. From one ensemble the prediction for the LHC $(\sqrt{s}=14 \mathrm{TeV})$ is $\sigma_{\text {tot }}^{p p}=113 \pm 5 \mathrm{mb}$ and from the other, $\sigma_{\text {tot }}^{p p}=140 \pm 7 \mathrm{mb}$. From each parametrization, and making use of derivative analyticity relations (DAR), we determine $\rho(s)$ (ratio between the forward real and imaginary parts of the elastic scattering amplitude). A discussion on the optimization of the DAR in terms of a free parameter is also presented. In all cases good descriptions of the experimental data are obtained.
\end{abstract}

\section{Introduction}

The total cross section, $\sigma_{\text {tot }}$, and the $\rho$ parameter (ratio of the real to imaginary part of the forward elastic scattering amplitude) are important quantities in the investigation of elastic hadron-hadron scattering at high energies $[1,2]$. They are expressed in terms of the elastic scattering amplitude, $F(s, t)$, by the formulas,

$$
\begin{gathered}
\sigma_{\text {tot }}(s)=4 \pi \operatorname{Im} F(s, t=0), \\
\rho(s)=\frac{\operatorname{Re} F(s, t=0)}{\operatorname{Im} F(s, t=0)},
\end{gathered}
$$

where $\sqrt{s}$ is the center-of-mass energy and $t$ the fourmomentum transfer squared.

For proton-proton ( $p p$ ) collisions, both quantities have been extracted from accelerator experiments and the results extend up to $\sqrt{s} \sim 63 \mathrm{GeV}$. On the other hand, $\sigma_{\text {tot }}^{p p}$ may also be inferred from cosmic-ray experiments at still higher energies, $\sqrt{s} \sim 10 \mathrm{TeV}$. However, these cosmic-ray results are model-dependent, since they are obtained from proton-air cross sections [3] and this has originated different results for $\sigma_{t o t}^{p p}$ in the interval $\sqrt{s}=5-25 \mathrm{TeV}$, which exhibit a reasonable degree of discrepancy.

Several models present extrapolations at the cosmicray region and the observed discrepancies may be ac- commodated by different models $[4,5,6,7,8]$. As we shall recall, presently, it is difficult to decide which could be the "correct" result and it seems that the general trend is to expect the new data from the next accelerator experiments, the BNL Relativistic Heavy Ion Collider (RHIC), $\sqrt{s} \sim 500 \mathrm{GeV}$ [9] and the CERN Large Hadron Collider (LHC), $\sqrt{s} \sim 14 \mathrm{TeV}[10]$.

However, at this stage, we understand that a model independent analysis of the experimental information presently available, taking detailed account of the discrepancies and its consequences, may bring important insights on the subject. This is the central point we are interested in.

In this communication, we first investigate two sets of distinct results for $\sigma_{t o t}^{p p}$, at cosmic-ray energies, in a model independent way that also takes into account of the experimental data at the accelerator region $(\sqrt{s}>10 \mathrm{GeV})$. In each case we fit four different analytic functions to each ensemble of data, using the CERN-MINUIT routine [11]. Next we make use of the one-subtracted derivative analyticity relation (DAR) in order to obtain analytic parametrizations for the $\rho$ parameter as function of the energy, from both ensembles and for all the parametrizations. In this investigation we stress that the general expression of the DAR has a free parameter and we present a study on the practical applicability of this parameter in the fits to the $\rho$ data. 
In all cases good descriptions of the experimental data are obtained.

The paper is organized as follows. In Sec. II we review the experimental information on $\sigma_{t o t}^{p p}$ from accelerator and cosmic-ray experiments and recall the main steps connecting integral and derivative analyticity relations. The fits concerning $\sigma_{t o t}^{p p}$ and the results for $\rho(s)$ are presented in Sec. III. In Sec. IV we discuss in some detail all the results obtained and present comparisons with some model predictions. The conclusions and some final remarks are the content of Sec. V.

\section{Experimental Information and Analytical Approach}

Here we first review the experimental information presently available on $\sigma_{t o t}^{p p}$ at the highest energies, stressing the puzzles involved at the cosmic-ray region. We also recall some essential formulas connecting integral and derivative analyticity relations and the corresponding derivative relation between $\sigma_{\text {tot }}^{p p}$ and $\rho$ (the analytical approach).

\section{II.1 Experimental information on $p p$ total cross sections}

As mentioned before, in order to analyze the experimental information presently available on $\sigma_{t o t}(s)$ for $p p$ interaction at energies beyond $\sim 10 \mathrm{GeV}$ (high energy region), we must distinguish between accelerator and cosmic-ray information. In the later case we follow a discussion first presented in Ref. [4].

Concerning accelerators, data on $\sigma_{\text {tot }}^{p p}$ from three experiments at the CERN Intersecting Storage Ring (ISR), between $23.5 \mathrm{GeV}$ and $62.3 \mathrm{GeV}$, were critically analyzed by Amaldi and Schubert and we shall consider here the final mean values from their analysis [12]. We also include the results at $13.8 \mathrm{GeV}$ and $19.4 \mathrm{GeV}$, obtained in Fermilab [12].

Although other accelerator data exist in this region, this set is suitable for the analyzes we are interested in. From one hand, these data represent the correct trend of $p p$ collisions in the region $10-60 \mathrm{GeV}$ and allow a statistical approach that includes different cosmic-ray information (see what follows). On the other hand, as will be discussed in Sec. IV, they are adequate for comparison with a model that predicts a faster rising of $\sigma_{t o t}^{p p}$ than generally expected.

Differently from accelerator data, cosmic-ray experimental information on $\sigma_{t o t}^{p p}$ comes from proton-air cross sections. We recall that antiprotons are not expected to have any significant role in these interactions and therefore the bulk of cosmic ray information on hadronnucleus cross sections does not concern antiprotons.

Now, since what is extracted in these experiments is the proton-air cross section, the determination of the $p p$ cross section depends on nuclear model assumptions $[3,4]$ and this has originated some puzzles, as reviewed in what follows.

The first information on $\sigma_{\text {tot }}^{p p}$ from cosmic ray experiments, at $\sqrt{s}=30 \mathrm{TeV}$, was reported by Baltrusaitis et al. in 1984 [13]. Extensive air showers recorded by Fly's Eye detector allowed the determination of the proton-air inelastic cross section. Based on Glauber theory, assuming a Gaussian profile for the nucleus and the proportionality between total cross section and the slope parameter (geometrical scaling), the authors inferred [13]

$$
\sigma_{\text {tot }}=120 \pm 15 \mathrm{mb} \text { at } \sqrt{s}=30 \mathrm{TeV}
$$

In 1987, based also on data from Fly's Eye experiment, Gaisser, Sukhatme and Yodh (GSY) introduced the limit $\sigma_{t o t}^{p p} \geq 130 \mathrm{mb}$ at $\sqrt{s} \sim 30 \mathrm{TeV}$ and, taking account of various process in the Glauber theory and additional assumptions, estimated [14]

$$
\sigma_{\text {tot }}^{p p}=175_{-27}^{+40} \mathrm{mb} \text { at } \sqrt{s}=40 \mathrm{TeV}
$$

Afterwards, extensive air shower data collected in the Akeno observatory allowed new estimates in the range $5-25 \mathrm{TeV}$, reported by Honda et al. [15], which is in agreement with the result reported by Baltrusaitis et al. and in disagreement with the GSY result. In particular, extrapolations by Honda et al. indicated [15]

$$
\sigma_{\text {tot }}^{p p}=133 \pm 10 \mathrm{mb} \text { at } \sqrt{s}=40 \mathrm{TeV}
$$

which is in agreement with the result reported by Baltrusaitis et al. and in disagreement with the GSY result. In the same year, Nikolaev claimed that the Akeno results have been underestimated by about $30 \mathrm{mb}$ [16] and, if we take this correction into account, the data in the interval $5-25 \mathrm{TeV}$ show agreement with the early GSY result. An important point is the fact that the analysis by Nikolaev seems correct, has never been criticized and the same is true for the GSY limit and result.

All these cosmic-ray informations are shown in Figure 1 , together with the accelerator data at lower energies. We stress that these experimental information concern only $p p$ and not $\bar{p} p$ interactions. From this Figure we clearly see the discrepancies between Honda/Baltrusaitis and GSY/Nikolaev.

For these reasons, in this paper we investigate the behavior of the total cross section taking account of the discrepancies that characterize the cosmic ray information. In order to do that, we consider two ensembles of data and experimental information, with the following notation: 
Ensemble A: accelerator data + Honda et al. + Baltrusaitis et al.

Ensemble B: accelerator data + Nikolaev + GSY

For ensemble $\mathrm{A}$ the references are $[12,13,15]$ and for ensemble B, $[12,14,16]$. In Sec. III we make use of different parametrizations to fit the data from each ensemble.

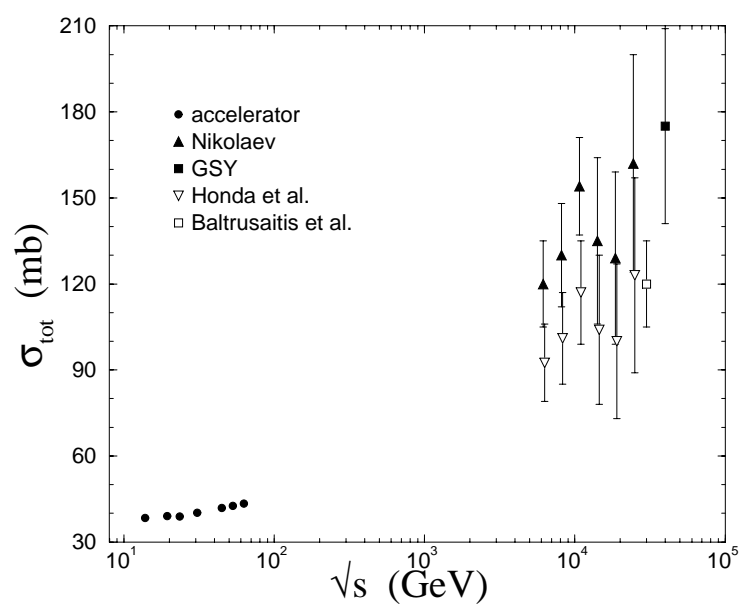

Figure 1. Experimental information on $p p$ total cross sections: accelerator data in the interval $13.8<\sqrt{s}<62.5 \mathrm{GeV}$ and cosmic-ray results in the interval $6.3 \mathrm{TeV}<\sqrt{s}<40$ $\mathrm{TeV}$.

\section{II.2 Derivative analyticity rela- tions and the $\rho$ parameter}

Both $\sigma_{t o t}(s)$ and $\rho(s)$ play a central role in the investigation of high-energy hadron scattering. Due to the connections between forward real and imaginary parts of the scattering amplitude, Eqs. (1) and (2), the analyticity (dispersion) relations constitute a suitable, model independent, approach for a simultaneous study of these quantities.

Although integral dispersion relations have been widely used in the study of hadronic scattering, in gen- eral, the analytical and/or numerical integrations are not an easy task. However, at sufficiently high energies, the smooth increase of $\sigma_{t o t}^{p p}$ (Fig. 1) allows to connect the integral form with a derivative one, which is easier to handle. The so called derivative analyticity relations (DAR) were introduced in the seventies [17] and since then have been critically investigated $[18,19]$. Recently, a recursive approach was developed, as well as generalizations to an arbitrary number of subtractions, for both cross even and odd amplitudes, near the forward direction [20]. As in the integral case, the convergences may be controlled by subtractions and specific formulas are associated with cross even and odd functions (scattering amplitudes in the case of particle-particle and anti particle-particle interactions) [2].

At this point we stress once more that we are only interested in $p p$ scattering (where discrepancies happen in $\left.\sigma_{t o t}(s)\right)$ and not $\bar{p} p$. Besides, our investigation concerns the highest energies, characterized by the smooth increase of $\sigma_{t o t}^{p p}$, nearly as a power on lns. For these reasons in our analyzes we will consider only an even amplitude, as the leading contribution, and only one subtraction.

A detailed deduction on how to obtain DAR from integral relations may be found in references $[17,18$, $19,20]$. Here we only review the main steps concerning our case of interest, namely, one subtraction and an even amplitude. We begin with the well known oncesubtracted integral dispersion relation (even amplitude) in the forward direction $(t=0)[2,21]$

$$
R e f_{+}(s)=K+\frac{2 s^{2}}{\pi} I,
$$

where $K \equiv \operatorname{Re} f_{+}(0)$ is the subtraction constant and

$$
I=P \int_{s_{0}}^{+\infty} d s^{\prime} \frac{1}{s^{\prime}\left(s^{\prime 2}-s^{2}\right)} \operatorname{Im} f_{+}\left(s^{\prime}\right)
$$

Following Bronzan, Kane and Sukhatme [17], and also [20], we consider a real parameter $\alpha$ so that after multiply and divide by $s^{\alpha}$ and integrating by parts we obtain

$$
\begin{aligned}
I & =\left.\frac{1}{2 s s^{\prime}} \ln \left|\frac{s^{\prime}-s}{s^{\prime}+s}\right| \operatorname{Im} f_{+}\left(s^{\prime}\right)\right|_{s^{\prime}=s_{0}} ^{\infty}- \\
& -\frac{1}{2 s} \int_{s_{0}}^{\infty} d s^{\prime} s^{\prime \alpha-1} \ln \left|\frac{s^{\prime}-s}{s^{\prime}+s}\right|\left(\frac{\alpha-1}{s^{\prime}}+\frac{d}{d s^{\prime}}\right) I m f_{+}\left(s^{\prime}\right) / s^{\prime \alpha}
\end{aligned}
$$

Taking account of the high-energy region $\left(s \gg s_{0} \sim m^{2} \sim 1 \mathrm{GeV}^{2}\right)$ and performing a change of variable $s^{\prime}=$ $e^{\xi^{\prime}}, \quad s=e^{\xi}$, the last equation may be put in the form

$$
I=\frac{1}{2 s} \int_{\ln s_{0}}^{\infty} d \xi^{\prime} s^{\prime \alpha-1} \ln \operatorname{coth} \frac{1}{2}\left|\xi-\xi^{\prime}\right|\left(\alpha-1+\frac{d}{d \xi^{\prime}}\right) I m f_{+}\left(s^{\prime}\right) / s^{\prime \alpha} .
$$


Expanding $\operatorname{Im} f_{+}\left(s^{\prime}\right) / s^{\prime \alpha}$ in powers of $\xi^{\prime}-\xi$, after some manipulation and taking account of the high energy limit $\left(s_{0} \rightarrow 0\right.$, that is, $\left.\ln s_{0} \rightarrow-\infty\right)$ we obtain

$$
R e f_{+}(s)=K+s^{\alpha} \sum_{n=0}^{\infty} \frac{d^{(n)}}{d \ln s^{(n)}}\left(I m f_{+}(s) / s^{\alpha}\right) \frac{I_{n}}{n !},
$$

where $I_{n}$ represents the integral in the variable $\xi^{\prime}$,

$$
I_{n}=\frac{1}{\pi} \int_{-\infty}^{+\infty} d \xi^{\prime} e^{(\alpha-1)\left(\xi^{\prime}-\xi\right)} \ln \operatorname{coth} \frac{1}{2}\left|\xi^{\prime}-\xi\right|\left(\alpha-1+\frac{d}{d \xi^{\prime}}\right)\left(\xi^{\prime}-\xi\right)^{n} .
$$

We have assumed that the series may be integrated term by term. Denoting $\xi^{\prime}-\xi \equiv y$ and integrating by parts, this equation may be put in the form

$$
I_{n}=\left.\frac{1}{\pi} \ln \operatorname{coth} \frac{1}{2}|y| e^{(\alpha-1) y} y^{n}\right|_{-\infty} ^{+\infty}+\frac{1}{\pi} \int_{-\infty}^{+\infty} d y \frac{e^{(\alpha-1) y}}{\sinh y} y^{n} .
$$

A central point here is that the first term on the right hand side converges to zero only for

$$
0<\alpha<2 \text {. }
$$

In this case we only have the second term, which can be expressed as a recursive relation in terms of the parameter $\alpha[20]$ :

$$
I_{n}=\frac{d^{(n)} I_{0}}{d \alpha^{(n)}}
$$

and integration in the complex plane gives

$$
I_{0}=\tan \left(\frac{\pi}{2}(\alpha-1)\right) .
$$

With this, we obtain an expression connecting the real part of an even amplitude with the derivatives of the imaginary part at the same energy, namely the DAR:

$$
\frac{\operatorname{Re} f_{+}(s)}{s^{\alpha}}=\frac{K}{s^{\alpha}}+\tan \left[\frac{\pi}{2}\left(\alpha-1+\frac{d}{d \ln s}\right)\right] \frac{\operatorname{Im} f_{+}(s)}{s^{\alpha}} .
$$

The leading term in the tangent series reads

$$
\tan \left(\frac{\pi}{2}(\alpha-1)\right) \frac{\operatorname{Im} f_{+}(s)}{s^{\alpha}}+\frac{\pi}{2} \sec ^{2}\left(\frac{\pi}{2}(\alpha-1)\right) \frac{d}{d \ln s}\left(\frac{\operatorname{Im} f_{+}(s)}{s^{\alpha}}\right),
$$

and making use of the normalization $\left(k^{2} \sim s\right)$,

$$
\frac{f_{+}(s)}{s} \equiv F(s, t=0),
$$

we obtain the general result for a forward amplitude:

$$
\begin{aligned}
& \operatorname{Re} F(s, 0)=\frac{K}{s}+\tan \left(\frac{\pi}{2}(\alpha-1)\right) \operatorname{Im} F(s, 0)+ \\
& +s^{\alpha-1} \frac{\pi}{2} \sec ^{2}\left(\frac{\pi}{2}(\alpha-1)\right) \frac{d}{d \ln s}\left(\frac{\operatorname{Im} F(s, 0)}{s^{\alpha-1}}\right) .
\end{aligned}
$$

At last, from equation (1), we obtain the general relation connecting $\rho$ and $\sigma_{t o t}$ :

$$
\rho(s)=\frac{4 \pi K}{s \sigma_{t o t}(s)}+\tan \left(\frac{\pi}{2}(\alpha-1)\right)+\frac{\pi}{2} \frac{s^{\alpha-1}}{\sigma_{t o t}(s)} \sec ^{2}\left(\frac{\pi}{2}(\alpha-1)\right) \frac{d}{d \ln s}\left(\frac{\sigma_{t o t}(s)}{s^{\alpha-1}}\right) .
$$


The standard form referred to in the literature and applied to hadronic scattering corresponds to the particular choice $\alpha=1$ (but usually without the subtraction constant) $[1,2,22,23]$, which we shall refer as the conventional form of the DAR:

$$
\rho(s)=\frac{4 \pi K}{s \sigma_{t o t}(s)}+\frac{1}{\sigma_{t o t}(s)} \frac{\pi}{2} \frac{d}{d \ln s}\left(\sigma_{t o t}(s)\right) .
$$

In the next section we use both the conventional and the general relations, in order to determine $\rho(s)$ from different parametrizations for $\sigma_{t o t}^{p p}(s)$ and ensembles of data.

\section{Fits and results}

In this section we first present the fits to the total cross sections for both ensembles defined in Sec. II and then the predictions for $\rho(s)$ obtained by means of DAR. The discussion on all the obtained results is the content of Sec. IV.

\section{III.1 Fits to total cross section}

For each ensemble defined in Sec. II, we fit the data with some standard and suitable parametrizations for the total cross sections:

$$
\text { fit 1: } \quad \sigma_{\text {tot }}=A+B \ln s+C(\ln s)^{2}
$$

$$
\text { fit 2: } \quad \sigma_{t o t}=A+B \ln s+C(\ln s)^{D}
$$

fit 3: $\quad \sigma_{\text {tot }}=A+B \ln s+C(\ln s)^{2}+R s^{-1 / 2}$

$$
\text { fit 4: } \quad \sigma_{\text {tot }}=A+B \ln s+C(\ln s)^{D}+R s^{-1 / 2}
$$

where $A, B, C, D$ and $R$ are free parameters.

The choice for these parametrizations was based on the following considerations. Firstly, since Figure 1 is a linear-log plot, we see that at the highest energies the data suggest an increase of $\sigma_{t o t}^{p p}$ as a polynomial on $l n s$. The Froissart-Martin bound states that the fastest rate permissible asymptotically for the rising of $\sigma_{t o t}^{p p}$ is $\ln ^{2} s$ [24]. However, it has been shown by the UA4/2 Collaboration that fits to $p p$ and $\bar{p} p$ accelerator data indicate the power $2.25_{-0.31}^{+0.35}$, a result referred to as a "qualitative saturation of the Froissart-Martin bound" [25]. For these reasons, we consider polynomial functions on $l n s$ with two possibilities for the power factor: the value 2, according to the asymptotical bound and as a free parameter to be determined by the fits. Finally, the power function on $s$, Eqs. (24) and (25), represents the usual way to take account of data at lower values of the energy $(5 \sim 20 \mathrm{GeV})$ and can be associated with Regge phenomenology.

The fits were performed by using the CERNMINUIT routine [11] and the results for ensembles A and $\mathrm{B}$ are shown in Figs. 2 and 3, respectively. The corresponding values of the free parameters and the $\chi^{2}$ per degree of freedom are displayed in Table 1 and 2 for ensembles A and B, respectively. We discuss all these results in Sec. IV.

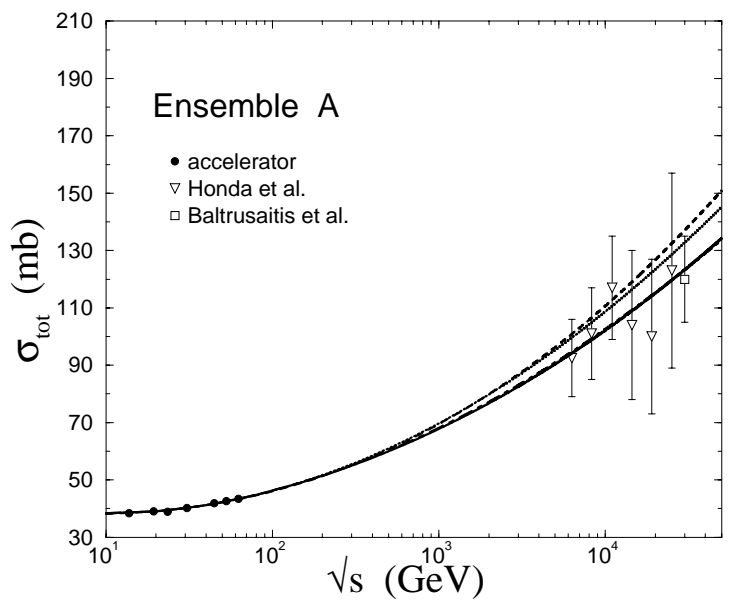

Figure 2. Fits to $p p$ total cross section data from ensemble A through Eqs. (22) - (25): fit 1 (solid), fit 2 (dotted), fit 3 (dot-dashed ) and fit 4 (dashed).

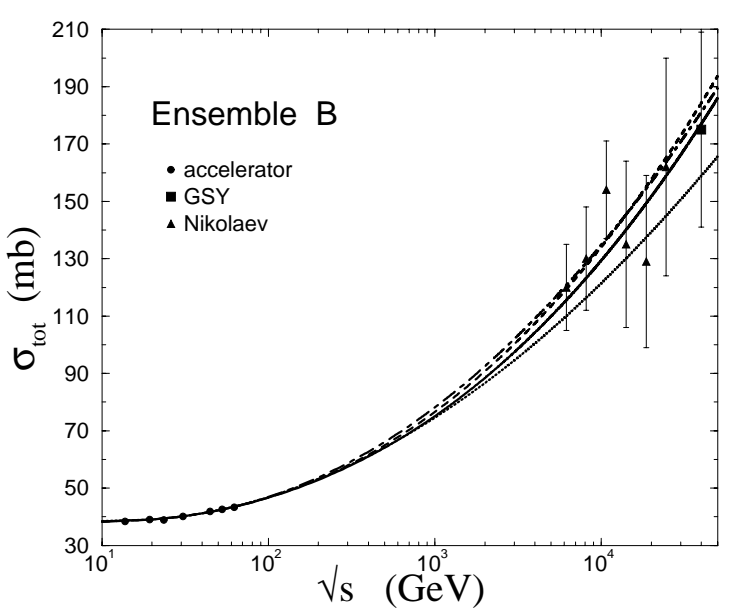

Figure 3. Fits to $p p$ cross section from Ensemble B. Same legend as Fig. 2. 
Table 1. Values of the parameters in Equations (22) (25) and the $\chi^{2}$ per degree of freedom in each fit to Ensemble A.

\begin{tabular}{ccccc}
\hline fit: & 1 & 2 & 3 & 4 \\
\hline$A$ & 45.78 & 42.64 & 38.74 & 33.67 \\
$B$ & -3.315 & -1.875 & -1.868 & -0.3201 \\
$C$ & 0.3654 & 0.1220 & 0.2894 & 0.09465 \\
$D$ & 2 (fixed) & 2.312 & 2 (fixed) & 2.288 \\
$R$ & - & - & 21.52 & 31.40 \\
$\chi^{2}$ & 11.7 & 15.4 & 9.8 & 9.9 \\
d.o.f. & 11 & 10 & 10 & 9 \\
$\chi^{2} /$ d.o.f. & 1.06 & 1.54 & 0.98 & 1.10 \\
\hline
\end{tabular}

Table 2. Values of the parameters in Equations (22) (25) and the $\chi^{2}$ per degree of freedom in each fit to Ensemble B.

\begin{tabular}{ccccc}
\hline fit: & 1 & 2 & 3 & 4 \\
\hline$A$ & 49.27 & 43.54 & 62.48 & 38.34 \\
$B$ & -4.435 & -1.846 & -7.221 & -0.8162 \\
$C$ & 0.4534 & 0.05958 & 0.6050 & 0.03461 \\
$D$ & 2 (fixed) & 2.624 & 2 (fixed) & 2.755 \\
$R$ & - & - & -38.40 & 14.80 \\
$\chi^{2}$ & 15.0 & 10.7 & 10.4 & 11.4 \\
d.o.f. & 11 & 10 & 10 & 9 \\
$\chi^{2} /$ d.o.f. & 1.36 & 1.07 & 1.04 & 1.27 \\
\hline
\end{tabular}

\section{III.2 Predictions for the $\rho$ param- eter}

In this section we determine the $\rho(s)$ behavior, making use of the DAR and all the parametrizations for $\sigma_{\text {tot }}^{p p}$ obtained in the last section. As commented before we are interested in the practical applicability of both forms of the DAR, the general and the conventional ones, Eq. (20) and Eq. (21), respectively.

In principle, the general expression (20) has two "free parameters", namely the subtraction constant $K$ and the parameter $\alpha$. Firstly, as a simple exercise to see the effect of the DAR, let us consider one of the fits to $\sigma_{t o t}^{p p}$, for example fit 4 , for both ensembles A and B, and calculate $\rho(s)$ through the conventional expression and without subtraction constant, which means taking $\alpha=1$ and $K=0$ in Eq. (20), or $K=0$ in Eq. (21) (this corresponds to the formula usually referred to in the literature $[1,2])$. The results are displayed in Fig. 4 together with the experimental data [26]. We see that although the predictions from both ensembles are similar at the ISR energy region, both disagree with the data.

In what follows we investigate the influence of the above two free parameters in the description of these data. In order to do that, we first treat the conventional formula with the subtraction constant and then the general formula, without subtraction constant and the factor $\alpha$ as free parameter.

- Conventional derivative dispersion relation

With the parametrizations (22-25) for $\sigma_{t o t}^{p p}$ we obtain analytical expressions for $\rho(s)$ by using the conventional form of the DAR, Eq. (21). For each input parametrization the subtraction constant $K$ is a free parameter, determined by fit, through the CERNMINUIT routine, to the experimental $\rho$ data [26].

The results from each parametrization and for both ensembles are shown in Figures 5 and 6 together with the experimental data. The values of the subtraction constant and statistical information about the fits are displayed in Table 3.

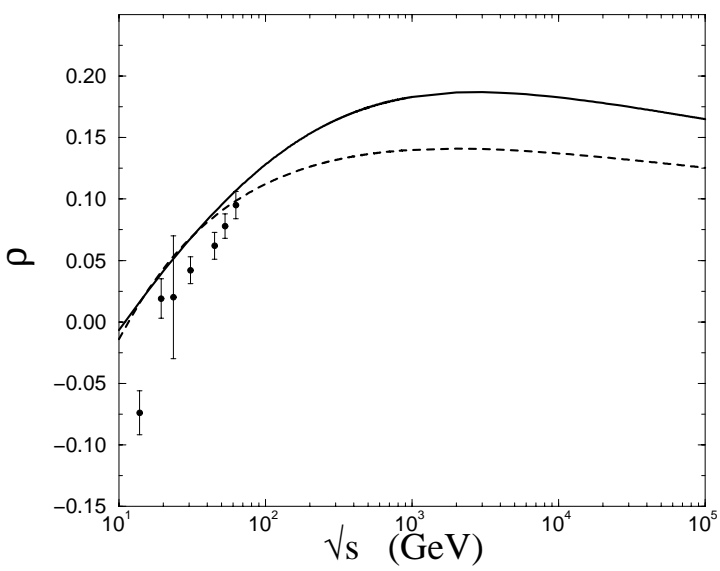

Figure 4. Results for $\rho(s)$, using fit 4 from both ensembles A (dashed) and B (solid) and DAR with $\alpha=1$ and $K=0$ in Eq. (20) and experimental data [26].

Table 3. Values of the subtraction constant from fits to $\rho(s)$ data and the $\chi^{2}$ for 6 degree of freedom. Calculation performed thorough Eq. (21) using the four fits to $\sigma_{\text {tot }}^{p p}$ from ensembles A and B.

\begin{tabular}{ccccc}
\hline fit- $\sigma_{\text {tot }}^{p p}-$ Ensemble A: & 1 & 2 & 3 & 4 \\
\hline$K$ & -134.1 & -135.6 & -130.3 & -129.6 \\
$\chi^{2} /$ d.o.f. & 0.82 & 0.63 & 0.97 & 0.63 \\
\hline fit- $\sigma_{\text {tot }}^{p p}-$ Ensemble B: & 1 & 2 & 3 & 4 \\
\hline$K$ & -130.0 & -134.4 & -137.1 & -132.0 \\
$\chi^{2} /$ d.o.f. & 2.0 & 1.48 & 1.85 & 1.44 \\
\hline \hline
\end{tabular}




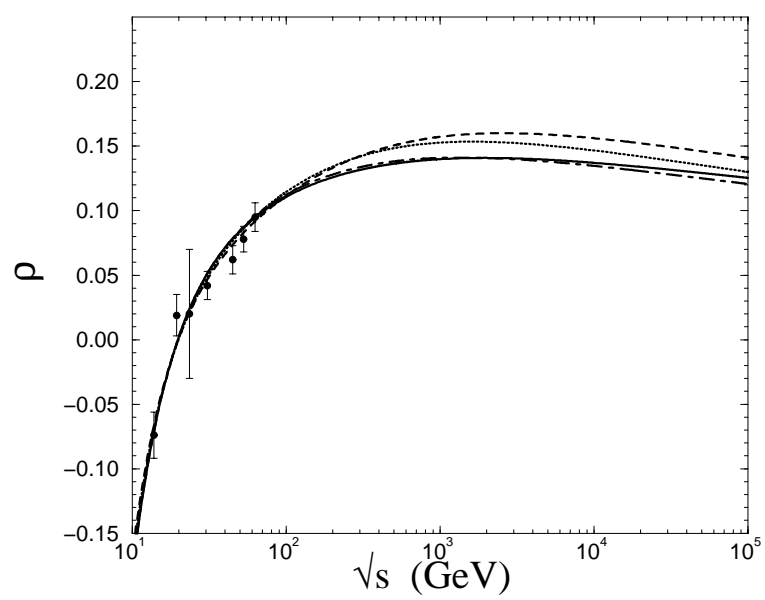

Figure 5. Results for $\rho(s)$, through the conventional DAR, Eq. (21), from fits to $\sigma_{\text {tot }}^{p p}$ from ensemble A together with the experimental data. Same legend as Fig. 2.

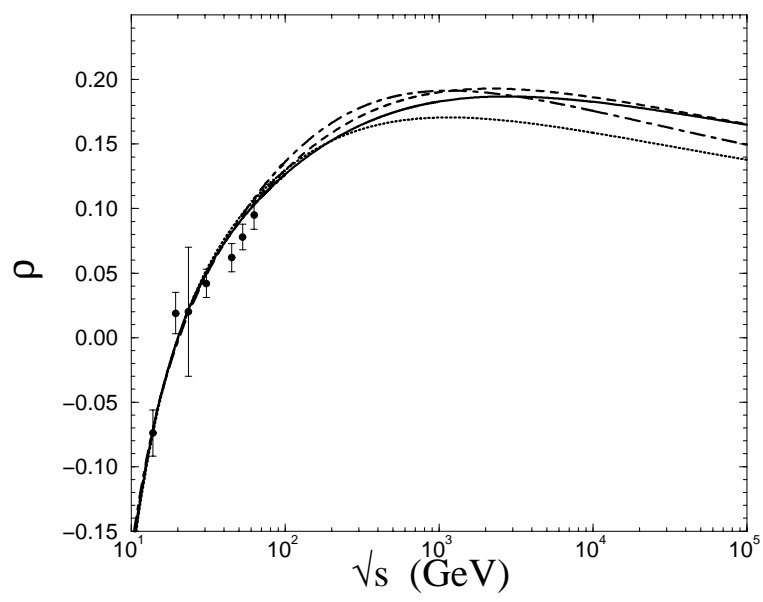

Figure 6. Results for $\rho(s)$, through the conventional DAR, Eq. (16), fits to $\sigma_{\text {tot }}^{p p}$ from ensemble B, together with the experimental data. Same legend as Fig. 2.

\section{- General derivative analyticity relation}

As shown in some detail in Sec. II.B, the general result for the DAR depends on the free parameter $\alpha$. It comes from the integration by parts of Eq. (7) and it is necessary in order to allow a finite derivative form associated with the integral form. In this sense it seems to play the role of a regularization factor. Although in nearly all practical uses of the DAR the value $\alpha=1$ is assumed, we have shown that its value is constrained to the interval $0<\alpha<2$ (see also references $[18,19]$ ). In particular, in the context of a multiple diffraction model, it was recently shown that the description of the experimental data on $p p$ elastic scattering may be improved by taking $\alpha$ as a free parameter [27]. This early result inspired us to make use of the general relation (20) and to investigate the possible effect of $\alpha$ as a free parameter (some preliminary results were already presented in Ref. [28]). To this end we will not take account of the subtraction constant in the general formula for the DAR, namely $K=0$ in Eq. (20), so that we explicitly have:

$$
\begin{aligned}
\rho(s) & =\tan \left(\frac{\pi}{2}(\alpha-1)\right)+ \\
& +\frac{\pi}{2} \sec ^{2}\left(\frac{\pi}{2}(\alpha-1)\right)\left[\frac{1}{\sigma_{t o t}(s)} \frac{d \sigma_{t o t}(s)}{d \ln s}+1-\alpha\right] .
\end{aligned}
$$

With a fixed parametrization for $\sigma_{t o t}^{p p}$ we can fit the experimental data on $\rho$ by letting $\alpha$ to be a free parameter in the above equation, once more by using the CERN-MINUIT. The results from all the parametrizations for $\sigma_{t o t}^{p p}$ with both ensembles A and B are shown in Figures 7 an 8, respectively. The corresponding values for $\alpha$ and statistical information about the fits are displayed in Table 4.

Table 4. Values of the parameter $\alpha$ from fits to $\rho(s)$ data and the $\chi^{2}$ for 6 degree of freedom. Calculation performed thorough Eq. (20) with $K=0$ and using the four fits to $\sigma_{t o t}^{p p}$ from ensembles A and B.

\begin{tabular}{ccccc}
\hline fit- $\sigma_{\text {tot }}^{p p}-$ Ensemble A: & 1 & 2 & 3 & 4 \\
\hline$\alpha$ & 1.229 & 1.223 & 1.231 & 1.230 \\
$\chi^{2} /$ d.o.f. & 2.61 & 3.17 & 2.17 & 2.20 \\
\hline fit- $\sigma_{\text {tot }}^{p p}-$ Ensemble B: & 1 & 2 & 3 & 4 \\
\hline$\alpha$ & 1.244 & 1.240 & 1.244 & 1.239 \\
$\chi^{2} /$ d.o.f. & 1.48 & 1.94 & 2.00 & 1.80 \\
\hline
\end{tabular}

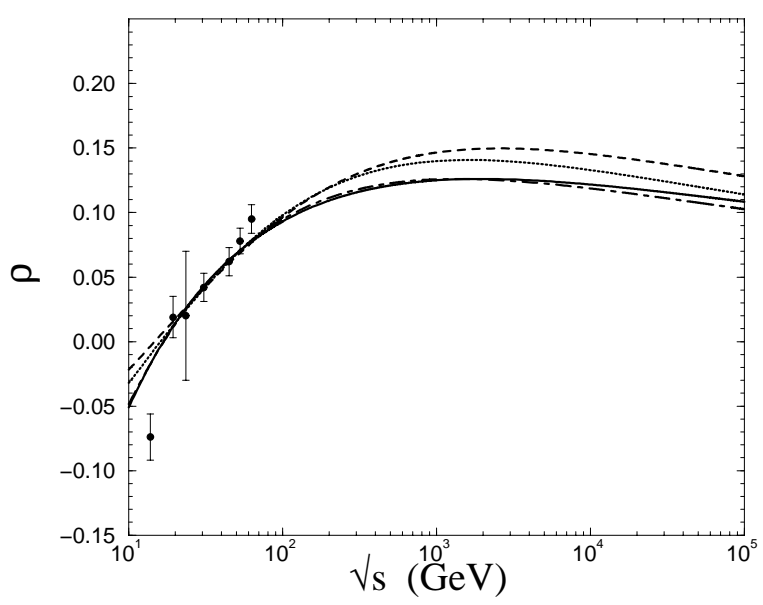

Figure 7. Results for $\rho(s)$, through the general expression of the DAR, Eq. (20), with $\alpha$ as free parameter, $K=0$ and fits to $\sigma_{\text {tot }}^{p p}$ from ensemble A. 


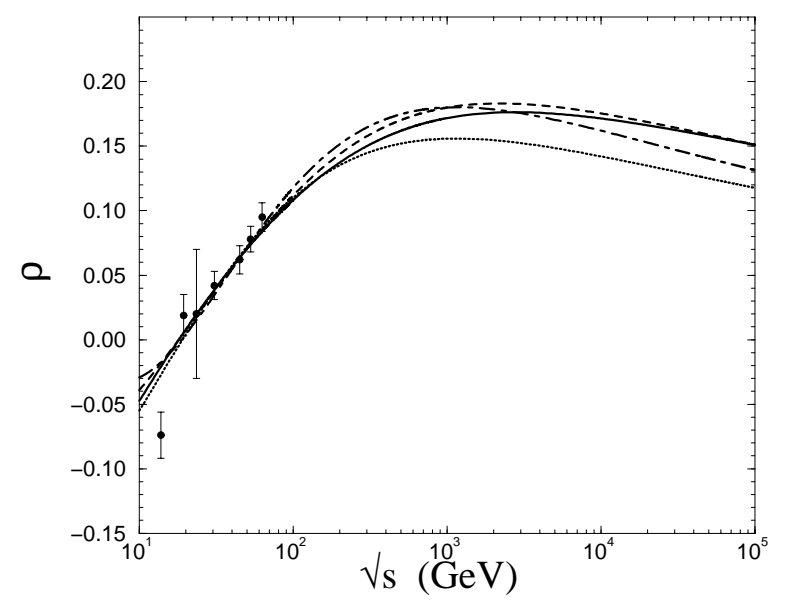

Figure 8. Results for $\rho(s)$, through the general expression of the DAR, Eq. (20), with $\alpha$ as free parameter, $K=0$ and fits to $\sigma_{\text {tot }}^{p p}$ from ensemble B.

\section{Discussion}

The parametrizations for $\sigma_{t o t}^{p p}$ are the usual ones, but the different ensembles, suggested by cosmic-ray results, introduce novel behaviors in the asymptotic region, as clearly shown in Figs. 2 and 3. For example, by calculating the average value and the standard deviation from the four parametrizations we can estimate $\sigma_{\text {tot }}^{p p}(14 \mathrm{TeV})=113 \pm 5 \mathrm{mb}$ for ensemble $\mathrm{A}$ and $\sigma_{\text {tot }}^{p p}(14 \mathrm{TeV})=140 \pm 7 \mathrm{mb}$ for ensemble B. At lower values of the energy, namely $\sqrt{s}$ in the region $10-100$ $\mathrm{GeV}$, there is no significant distinction between the four parametrizations. In general, model predictions are in agreement with the above result from ensemble $\mathrm{A}$ $[5,6,7]$, including the fit by the UA4/2 Collaboration [25]. To our knowledge, the only exception (model) that presents agreement with the result from ensemble $\mathrm{B}$ is that of Ref. [4]. This multiple diffraction model is based on analyzes of $p p$ elastic scattering in the interval $13.8 \mathrm{GeV} \leq \sqrt{s} \leq 62.5 \mathrm{GeV}$, the same set we used here at the accelerator region. Extrapolation to higher energies predicted $\sigma_{\text {tot }}(16 \mathrm{TeV})=147 \mathrm{mb}$, without estimated errors. Recently Pérez-Peraza et al. improved this model predictions determining confident error bands through a forecasting regression analysis [29]. Reading from Fig. 2 of this reference we can infer $\sigma_{t o t}(16 \mathrm{TeV}) \sim 147 \pm 37 \mathrm{mb}$. Despite of the large error band ( $\sim 25 \%$ ), even in this case it is clear from the quoted figure that the results favors ensemble B. From a "statistical point of view" we may say that the published results from models and fits show agreement with ensemble A. However, it should be stressed that the cosmic-ray estimations in the ensemble A were obtained under the hypothesis of the geometrical scaling [3], which is violated even at $\sqrt{s} \sim 500 \mathrm{GeV}$. Moreover, we should remember results from cosmic-ray experiments which indicate the possibility of new phenomena in $p p$ collisions at center-of-mass energies beyond $500 \mathrm{GeV}$ [30]. As a direct consequence we should expect new open channels and therefore a faster rising of the $p p$ total cross section than expected in the extrapolations from accelerator data. This seems to be well accommodated by the parametrizations with ensemble B.

Concerning the determination of $\rho(s)$ through DAR, we first showed that the conventional expression without subtraction constant $(\alpha=1$ and $K=0)$ does not reproduce the experimental $\rho$ data (Fig. 4). We recall that this expression has been referred to in the literature $[1,2]$ and also used in the context of phenomenological models [22, 23]. Taking account of the subtraction constant $K$, the data are well described, as shown in Figs. 5 and 6. From Table 3,

$$
|K| \sim 130-137,
$$

depending on the fit and ensemble for $\sigma_{t o t}^{p p}$. As expected different ensembles correspond to distinct behaviors at the highest and asymptotic energies. As in the case of $\sigma_{\text {tot }}^{p p}$ we can estimate an average value and standard deviation from the four results in Figs. 5 and 6: $\rho(14 \mathrm{TeV})=0.142 \pm 0.010$ with $\sigma_{\text {tot }}^{p p}$ from ensemble $\mathrm{A}$ and $\rho(14 \mathrm{TeV})=0.173 \pm 0.013$ from ensemble B. In this case we can say that the results with ensemble $\mathrm{A}$ present the best agreement with the experimental data (Table 3 and Figs. 5 and 6). We also tested the general expression for the DAR by taking $K=0$ and letting $\alpha$ as a free fit parameter. The results presented in Figs. 7 and 8 show that the data are also satisfactorily described, specially in the case of $\sigma_{\text {tot }}^{p p}$ from ensemble B (compare with Fig. 4, the case of fixed $\alpha=1$ ). From Table 4,

$$
\alpha \sim 1.22-1.24,
$$

depending also on the fit and ensemble for $\sigma_{t o t}^{p p}$. In all these cases the condition (13) is verified.

\section{Conclusions and final remarks}

In this communication we investigated two ensembles of experimental information on $p p$ total cross sections and used four different parametrizations to fit the data, as function of the energy. In each case we obtained predictions for $\rho(s)$ making use of both the conventional and the general expressions for the derivative analyticity relations.

Our first main conclusion is that experimental information presently available on $p p$ total cross sections indicates two possible different scenarios for the hadronic interactions at the highest energies. The fast rise of $\sigma_{\text {tot }}^{p p}$ from the analysis of ensemble B is corroborated by the multiple diffraction model of Ref. [4] and also 
indication of new phenomena from emulsion chamber experiments [30]. Although in this work we only point out for this possibility, new information coming from RHIC and LHC shall certaintly clarify this subject. In this sense, our results may be viewed as a kind of warning against some possible precipitated assumptions, namely, that extrapolations from accelerator data which show agreement with the cosmic-ray estimations in ensemble A could be the final answer to the question.

A second novel result from this model independent analysis was to show the practical applicability of both the conventional and the general expressions for the derivative analyticity relations at sufficiently high energies. In the conventional case, $\alpha=1$, letting the constant $K$ as free parameter, the description of the experimental $\rho$ data is quite good. On the other hand, taking $K=0$ and letting $\alpha$ as free fit parameter, the results may be considered satisfactory. In both cases the predictions at the highest energies are practically the same (compare Figs. 5 and 7 and also Figs. 6 and and 8) and the differences at this energy region come obviously from the different ensembles for $\sigma_{t o t}^{p p}$. Our approach was to consider the two possibilities separately ( $K \neq 0$ or $\alpha \neq 1$ ), so that we could infer the intervals of possible variations, Eqs. (27) and (28). Simultaneous analysis with both possibilities shall improve the description of $\rho(s)$ and this is our second main conclusion.

In this communication we treated only $p p$ interactions, since the cosmic-ray informations concern only this case. We are presently investigating the inclusion of antiproton-proton data in the analysis through adequate considerations on crossing symmetry.

We are grateful to Fapesp for financial support and J. Montanha for reading the manuscript. M.J.M. is also thankful to N.N. Nikolaev for discussions, V. Ezhela for correspondence and $\mathrm{CNPq}$ for financial support.

\section{References}

[1] G. Matthiae, Rep. Prog. Phys. 57, 743 (1994).

[2] M. M. Block and R. N. Cahn, Rev. Mod. Phys. 57, 563 (1985).

[3] R. Engel, T. K. Gaisser, P. Lipari, and T. Stanev, Phys. Rev. D 58, 014019 (1998).

[4] A. F. Martini and M. J. Menon, Phys. Rev. D56, 4338 (1997).

[5] A. Donnachie and P. V. Landshoff, Z. Phys. C 2, 55 (1979); Phys. Lett. B 387, 637 (1996).

[6] P. Desgrolard, M. Giffon and E. Predazzi, Z. Phys. 63, 241 (1994).

[7] C. Bourrely, J. Soffer and T. T. Wu, Nucl. Phys. B 247, 15 (1984); Z. Phys. C 37, 369 (1988).

[8] M. M. Block, F. Halzen, T. Stanev, Phys. Rev. Lett. 83, 4926 (1999).
[9] The PP2PP (R2) Collaboration, BNL, www.rhic.bnl.gov.

[10] The TOTEM Collaboration, Technical proposal, CERN/LHCC 99-7, (1999).

[11] F. James, MINUIT - Function Minimization and Error Analysis - Reference Manual, Version 94.1, CERN D506 (1994).

[12] Accelerator data on $\sigma_{\text {tot }}^{p p}$ (the numbers between the squared brackets indicate the center-of-mass energy in GeV): A.S. Carrol et al., Phys. Lett. B61, 303 (1976) [13.8]; A.S. Carrol et al., Phys. Lett. B80, 423 (1979) [19.4]; U. Amaldi and K. R. Schubert, Nucl. Phys. B166, 301 (1980) [23.5, 30.7, 44.7, 52.8, 62.5].

[13] R. M. Baltrusaitis et al., Phys Rev. Lett. 52, 1380 (1984).

[14] T.K. Gaisser, U.P. Sukhatme, and G.B. Yodh, Phys. Rev. D36, 1350 (1987).

[15] H. Honda et al. (Akeno Collaboration), Phys. Rev. Lett. 70, 525 (1993).

[16] N.N. Nikolaev, Phys. Rev. D48, R1904 (1993).

[17] N.V. Gribov and A.A. Migdal, Sov J. Nucl. Phys. 8, 583 (1969); J. B. Bronzan, Argonne Symposium on the Pomeron, ANL/HEP 7327 (1973) 33; J. D. Jackson, 1973 Scottish Summer School, LBL-2079 (1973) 39; J. B. Brozan, G. L. Kane, and U. P. Sukhatme, Phys. Lett. B49, 272 (1974); K. Kang and B. Nicolescu, Phys. Rev. D11, 2461 (1975).

[18] G. K. Eichmann and J. Dronkers, Phys. Lett. B 52, 428 (1974); J. Heidrich and E. Kazes, Lettere al Nuovo Cimento 12, 365 (1975); A. Bujak and O. Dumbrajs, J. Phys. G: Nucl. Phys. 12, L129 (1976).

[19] J. Fischer and P. Kolár, Phys. Lett. B 64, 45 (1976); Phys. Rev. D 17, 2168 (1978); Czech. J. Phys. B 37, 297 (1987); P. Kolár and J. Fischer, J. Math. Phys. 25, 2538 (1984).

[20] M. J. Menon, A. E. Motter, and B. M. Pimentel, Phys. Lett. B 451, 207 (1999); in 1997 - XVIII Brazilian National Meeting on Particles and Fields, edited by O. J. P. Éboli et al. (SBF, São Paulo, 1998) pp. 548-552.

[21] F.W. Byron, R.W. Fuller, Mathematics of Classical and Quantum Physics, (Dover, 1992), Chap. 6.

[22] U. Sukhatme et al., Phys. Rev. D 12, 3431 (1975); V. Barger, J. Luthie and R. J. N. Phillips, Nucl. Phys. B 88, 237 (1975).

[23] M. Kawasaki, T. Maehara, M. Yonezawa, Phys. Lett. B 348, 623 (1995)

[24] M. Froissart, Phys. Rev. 123, 1053 (1961); A. Martin, and F. Cheung, Analytic Properties and Bounds of the Scattering Amplitude, (Gordon and Beach, New York, 1970).

[25] C. Augier et al. (UA4/2 Collaboration), Phys. Lett. B 315, 503 (1993).

[26] Experimental data on $\rho(s)$ (the numbers between the squared brakets indicate the $\mathrm{cm}$ energy in $\mathrm{GeV})$ : L. A. Fajardo et al. Phys. Rev. D24, 46 (1981) [13.8 and 19.4]; U. Amaldi and K. R. Schubert, Nucl. Phys. B166, 301 (1980) $[23.5,30.7,44.7,52.8,62.5]$. 
[27] A.F. Martini, M.J. Menon, J.T.S. Paes e M.J. Silva Neto, Phys. Rev. D59, 116006 (1999).

[28] R. F. Ávila, E. G. S. Luna, and M. J. Menon, in Proc. XXI Brazilian National Meeting on Particles and Fields, São Lourenço, Brazil, 23 - 27 October, 2000 (in press).
[29] J. Pérez-Peraza et al., hep-ph/0011167 (2000).

[30] Chacaltaya and Pamir Collaboration, Nucl. Phys. B 370, 365 (1992); S. L. C. Barroso et al., Nucl. Phys. B (Proc. Supp.) A75, 150 (1999). 